\title{
OLINK
}

$B|O S C| E N C E$

\section{Highly specific detection of phosphorylated proteins by Duolink}

\section{Olink Bioscience has developed a new protein detection assay that allows precise detection and quantification of proteins, protein interactions and protein modifications in fixed cells and tissue samples. The Duolink $k^{\circledast}$ immunoassay reagents are based on the in situ proximity ligation assay, capable of reporting proteins as countable distinct fluorescent or chromogenic spots visualized by standard microscopy. The assay exhibits a very high specificity for detection of phosphorylated proteins owing to a dual recognition format.}

Protein phosphorylation has a central role in important cellular events, such as signal transduction and cell cycle progression, emphasizing the importance of highly specific protein phosphorylation analysis. To allow detailed studies of protein phosphorylation, we have developed Duolink $^{\circledR}$, a product based on in situ PLA ${ }^{\mathrm{TM}}$. Duolink makes it possible to visualize and quantify specific protein phosphorylation events in situ with unprecedented specificity.

The most common techniques for detecting and quantifying protein phosphorylation today are immunoprecipitation and western blotting. Both techniques require large numbers of the cells of interest, which are difficult to obtain for stem cells and tissue material. Moreover, cell lysis, required in both approaches, does not maintain the subcellular localization of phosphorylated proteins.

Standard in situ protein detection protocols such as immunofluorescence and immunohistochemistry involve the use of only a single primary antibody recognizing its target protein. Phosphospecific antibodies, however, often recognize phosphorylated epitopes of closely related targets. Duolink, a kit series enabling the use of two primary antibodies for in situ immunoassays, brings the element of dual recognition to localized analyses. This makes it possible to use one antibody to a receptor and another antibody to a phosphorylation site on the same receptor. Using Duolink, a signal is only generated when the two antibodies are bound in close proximity, such as on the same receptor molecule or in the same complex.

\section{The Duolink reagents and in situ PLA technology}

The Duolink user first applies two primary antibodies selected from two different host species - such as one mouse and one rabbit antibody - to fixed cells or tissue sections, followed by addition of generic

\section{Mats Gullberg \& Ann-Catrin Andersson}

Olink AB, Uppsala, Sweden. Correspondence should be addressed to M.G. (mats.gullberg@olink.com).
Duolink secondary species-specific antibodies containing unique DNA strands. Added oligonucleotides that hybridize to the DNA strands on the secondary antibodies form a circular template upon addition of ligase and when in close proximity $(<40 \mathrm{~nm})^{1-3}$. This template, still anchored to the antibody, is subsequently amplified, and the products are detected using complementary, labeled oligonucleotide probes. The resulting distinct spots are derived from single-molecule proteinphosphorylation events (Fig. 1).

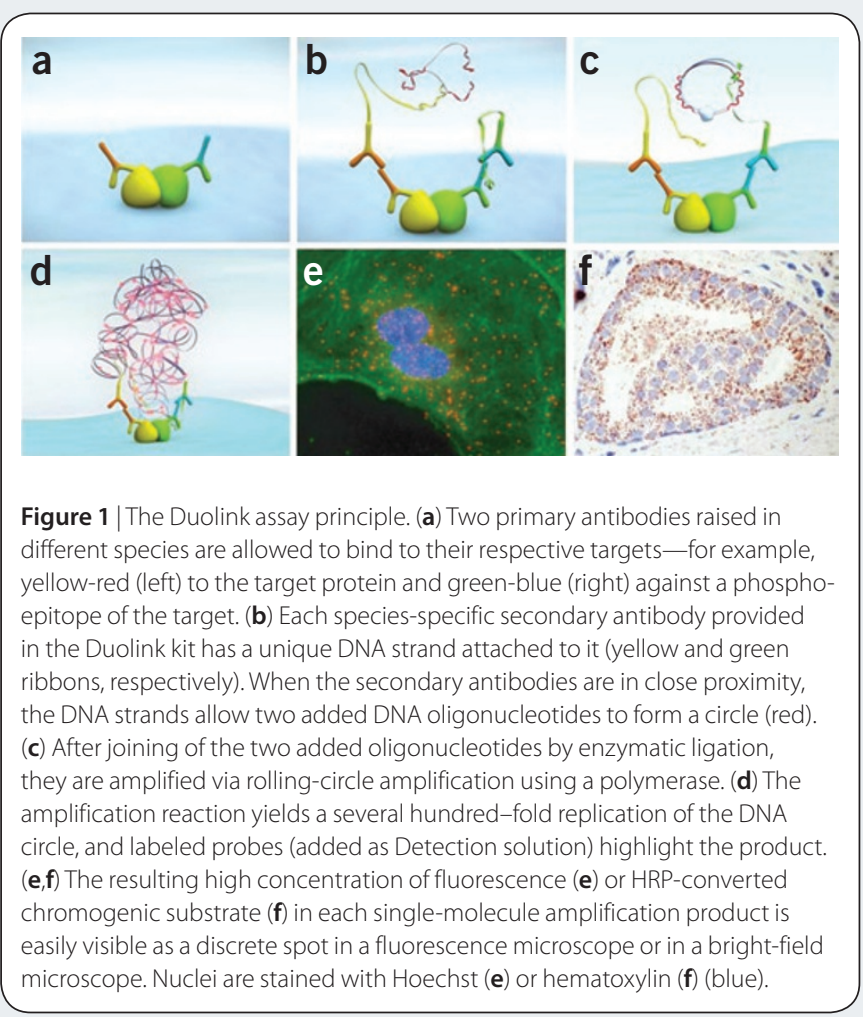



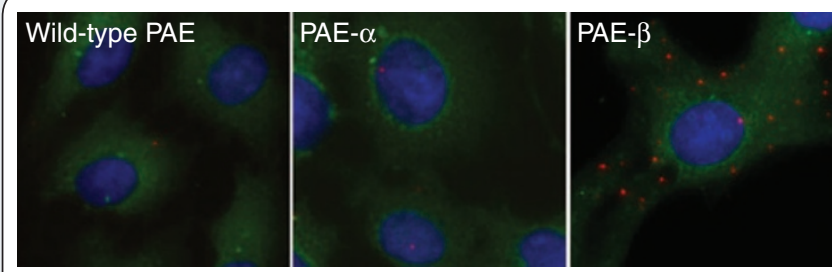

Figure $\mathbf{2}$ | Visualization of phosphorylated PDGFR- $\beta$. Shown are images of wildtype PAE cells in the native state (left), PDGF-BB-stimulated PAE-a cells (middle) and PDGF-BB-stimulated PAE- $\beta$ cells (right) visualized using one antibody to PDGFR- $\beta$ and one antibody to P(Tyr751)-PDGFR- $\beta$ to specifically detect PDGFR- $\beta$ phosphorylated in PAE- $\beta$ cells. Each red dot represents an activated receptor, detected by in situ PLA ${ }^{\mathrm{TM}}$ using the Duolink ${ }^{\circledR}$ kit. Nuclei are stained with Hoechst 33342 (blue) and microtubules with a FITC-conjugated antibody to a-tubulin (green).

\section{Duolink for highly specific phosphorylation detection}

When investigating signaling pathways and protein activation, one often stimulates the cells to promote the activation, as illustrated by platelet-derived growth factor (PDGF)-BB stimulation of different porcine aortic endothelial (PAE) cells (Fig. 2). Wild-type PAE cells do not express human PDGFR, PAE- $\alpha$ cells express human PDGFR- $\alpha$, and PAE- $\beta$ cells express human PDGFR- $\beta$. Duolink reagents and two primary antibodies, one targeting human PDGFR- $\beta$ and one targeting human P(Tyr751) PDGFR- $\beta$, correctly revealed strong signals only in PAE- $\beta$ cells.

The advantage of dual recognition for specific detection of phosphorylated PDGFR- $\beta$ is evident in a comparison of data obtained using Duolink, requiring two antibodies to elicit a signal, with a traditional assay using only one phosphospecific antibody. A clear discrimination between signal generated in PAE- $\beta$ cells over signal obtained in PAE- $\alpha$ or wild-type PAE cells was obtained using Duolink (Fig. 3a) compared to data obtained using only one phosphospecific antibody (Fig. 3b).

\section{Pan-specific antibodies and Duolink}

In situations in which there are no available antibodies directed against a desired modification, Duolink allows to the use of a pan-specific antibody together with a target-specific antibody. An excellent example of this was recently published, implementing detection of phosphorylated ninein ${ }^{4}$, a protein involved in positioning and anchorage of the microtubule in epithelial cells, by combining a pan-specific antibody to phosphotyrosine with an antibody to ninein.

\section{Phosphorylation-interaction detection}

Duolink allows not only highly specific detection of phosphorylation events but also detection of interactions between two proteins. This makes it possible to determine which interactions will lead to activation of which phosphorylation site on a particular receptor. It also holds great promise for studying activated receptors, homo- and heterodimeric, in cases in which dimers can be present without being activated, as speculated for the Her family of receptors ${ }^{5}$.
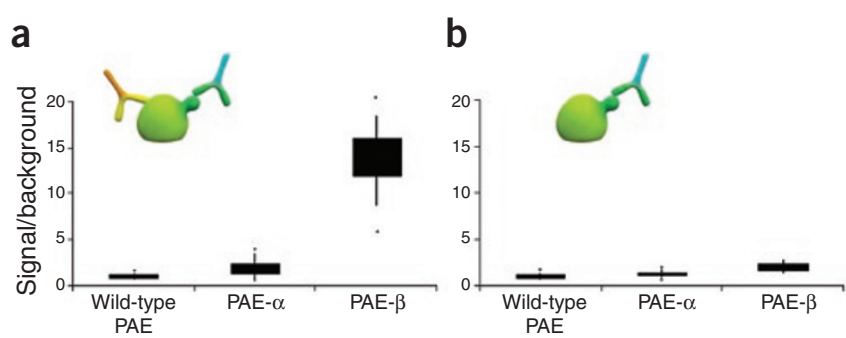

Figure 3 | Highly selective detection of phosphorylated PDGFR- $\beta$ using Duolink compared to a standard, single-binder immunocytochemical assay. (a,b) Schematics of Duolink double recognition (a) and a single primary antibody to P(Y751) PDGFR- $\beta$ (b), which is equivalent to a standard immunofluorescence assay, but developed using Duolink reagents to generate comparable quantifiable signals. The graphs are box-plot representations of the ratios between signals obtained in the different cell types over the average signal obtained in wild-type PAE cells (negative control). PAE- $\alpha$ and PAE- $\beta$ cells were stimulated with PDGF $B B$ before fixation. Wild-type PAE and PAE-a should be negative as they do not express human PDGFR- $\beta$, whereas an increase in signal should be seen in PAE- $\beta$ cells $(n=20$ image fields with $\sim 20$ cells per image per sample type, each sample run in duplicate. The box represents the distribution of the data points, stretching from the 25th percentile to the 75th percentile. The lengths of the lines above and below the box are defined by the maximum and minimum data point values, respectively. Outlying data points that do not fall within 2 s.d. of the median value are shown as dots.

\section{Conclusions}

In situ PLA, using the Duolink reagents, provides a powerful yet simple means to quantitatively visualize post-translational modifications such as phosphorylation of proteins in fixed cells and tissue with exceptional specificity and sensitivity. In addition, covalently modified, such as ubiqutinylated and SUMOylated, proteins can also be quantified in situ because of the dual recognition format provided by Duolink. There is no need to overexpress target proteins, and data interpretation is facilitated by countable spots, each representing a single-molecule event. The Duolink reagents are available to multiple species of primary antibodies to fit the unique application of the user.

Published specific Duolink assays, as well as assays developed with partners — for example, Abnova antibody pairs for PLA—are listed with relevant references in a searchable assay database at http://www.olink. $\mathrm{com} /$.

1. Fredriksson, S. et al. Protein detection using a proximity-dependent DNA ligation assay. Nat. Biotechnol. 20,473-477 (2002).

2. Söderberg, O. et al. Direct observation of individual endogenous protein complexes in situ by proximity ligation. Nat. Methods 3, 995-1000 (2006).

3. Jarvius, M. et al. In situ detection of phosphorylated platelet-derived growth factor receptor $\beta$ using a generalized proximity ligation method. Mol. Cell. Proteomics $\mathbf{6}$, 1500-1509 (2007).

4. Matsumoto, T. et al. Ninein is expressed in the cytoplasm of angiogenic tip-cells and regulates tubular morphogenesis of endothelial cells. Arterioscler. Thromb. Vasc. Biol. 28, 2123-2130 (2008)

5. Tao, R.-H. \& Maruyama, I.N. All EGF(ErbB) receptors have preformed homo- and heterodimeric structures in living cells. J. Cell Sci. 121, 3207-3217 (2008).

This article was submitted to Nature Methods by a commercial organization and has not been peer reviewed. Nature Methods takes no responsibility for the accuracy or otherwise of the information provided. 